\title{
General flow of fluid into a drainage slit on an impervious base
}

\author{
S. K. JAIN $\left({ }^{*}\right)$ \\ Received on November 5, 1981
}

\section{ABSTRACT}

In steady state conditions, two-dimensional general flow of an incompressible fluid through undeformable porous media, consistent with specific boundary conditions, is considered. The general equation of the free surface is obtained, and is illustrated by means of a numerical example.

\section{SUMMARY}

In the present paper, a steady state two-dimensional general flow of an incompressible fluid through undeformable porous media is considered. The flow is characterised by a non-linear partial differential equation

$$
\frac{\partial}{\partial v}\left(\frac{v}{F} \cdot \frac{\partial H}{\partial v}\right)+\frac{1}{F}\left(\frac{1}{v}+\frac{F^{\prime}}{F}\right) \frac{\partial^{2} H}{\partial \theta^{2}}-\underline{\Omega},
$$

where $H$ denotes the total head, $\theta$ the angle between the velocity vector $\vec{v}$ and the $x$-axis and $F^{\prime}$ stands for the first order derivative of $F$ with respect to $v$. The specific boundary conditions consistent with the flow system are:

(i) laminar at the head reservoir, that is $H=H_{0}, \theta=0$,

(ii) turbulent at the drainage slit, that is $H=0, \theta=\frac{\pi}{2}$, and

(iii) at the straight stream line $\mathrm{OX}, \frac{\partial H}{\partial \theta}-=0, \theta=0$.

The general equation of the free surface is obtained employing Frobenius method of series solution and is illustrated by means of a numerical example.

(*) Department of Mathematics, Rivers State College of Education, Rumuolumeni, P. M. B. 5047, PORT HARCOURT (NIGERIA). 


\section{RIASSUNTO}

Viene preso in considerazione in condizioni stazionarie un flusso generale bidimensionale di un fluido incompressibile attraverso mezzi porosi indeformabili, con specifiche condizioni al contorno. $E^{\prime}$ stata ottenuta l'equazione generale della superficie libera, ed essa viene illustrata da esempi numerici.

\section{1. - INTRODUCTION}

As the nature of flow mainly depends upon the fluid velocity and the structural constitution of the porous matrix through which it flows, the consideration of a single type of natural flow of fluid through porous media is an ideal situation which is hardly possible. Therefore, the assumption of general flow (Bear, 1972) which includes in it the consideration of mainly two types of flow, namely laminar and turbulent, appears to be more practicable. In its passage of transition from laminar to turbulent, the flow is termed as non-linear laminar which takes place under the predominance of inertial forces. Consequently, Elenbaas and Katz (1948), Engelund (1953), Jain and Upadhyay (1976), and Upadhvay $(1975,1977)$ obtained specific solutions of certain nonlinear laminar and turbulent flow problems.

In the present paper, we consider a steady state two-dimensional general flow of an incompressible fluid through undeformable porous media. The flow is characterized by a non-linear partial differential equation with specific boundary conditions consistent with the flow system. The nature of the free surface is obtained employing Frobenius (Davis, 1952) method of series solution.

\section{2. - EQuATION OF FLUID FLOW IN POROUS MEDIUM}

Assuming that the flow is linear upto a critical Reynolds number $\hat{\epsilon}_{r}$ and non-linear beyond it, we have the general law of 
fluid flow through a porous medium in the form (Scheidegger, 1960),

$$
-\operatorname{grad} H=F(v) \overrightarrow{\mathrm{v}}
$$

where

$$
\begin{aligned}
& F(v)=\frac{1}{n}, \xi \leqslant \xi_{c}, \\
& \vec{v}=a+b v, \xi \leqslant \xi_{c} .
\end{aligned}
$$

It is evident from [1] that for $\dot{\zeta}>\bar{\xi}_{c}$, there are two specific flows-nonlinear laminar and turbulent according whether $a \neq 0$ or $a=0$ respectively, since the constant $" a$ " signifies the effect of linearity in the law.

The non-linear partial differential equation governing the two-dimensional general flow in steady state condition is

$$
\frac{\partial}{\partial v}\left(\frac{v}{F} \cdot \frac{\partial H}{\partial v}\right)+\frac{1}{F}\left(\frac{1}{v}+\frac{F^{\prime}}{F}\right) \frac{\partial^{2} H}{\partial \theta^{2}}=0,
$$

where $H$ denotes the total head, $\theta$ the angle between the velocity vector $\overrightarrow{\mathrm{v}}$ and the $x$-axis and $F^{\prime}$ stands for the first order derivative of $F$ with respect to $v$. While obtaining the equation [2], it has been assumed that the flowing fluid and the fluid bearing strata are both incompressible.

\section{3. - Formulation of the PROBLEM}

In steady state condition, we consider the general flow of an incompressible fluid moving through an infinitely extended undeformable porous medium and running into the drainage slit OP (cf. Fig. 1). The slit is assumed to be a permeable surface on a horizontal impervious stratum OX. In the present problem, we take the flow as (i) laminar at the head reservoir, where $H=H_{0}$ and $\theta=0$, and (ii) turbulent at the drainage slit at which $H=0$ 
and $\theta=\frac{\pi}{2}$. Besides the boundary conditions (i) and (ii), we have along the straight stream line $\mathrm{OX}, \frac{\partial H}{\partial \theta}=0$ and $\theta=0$.

The problem is to determine the general equation of the free surface corresponding to the aforesaid flow system.

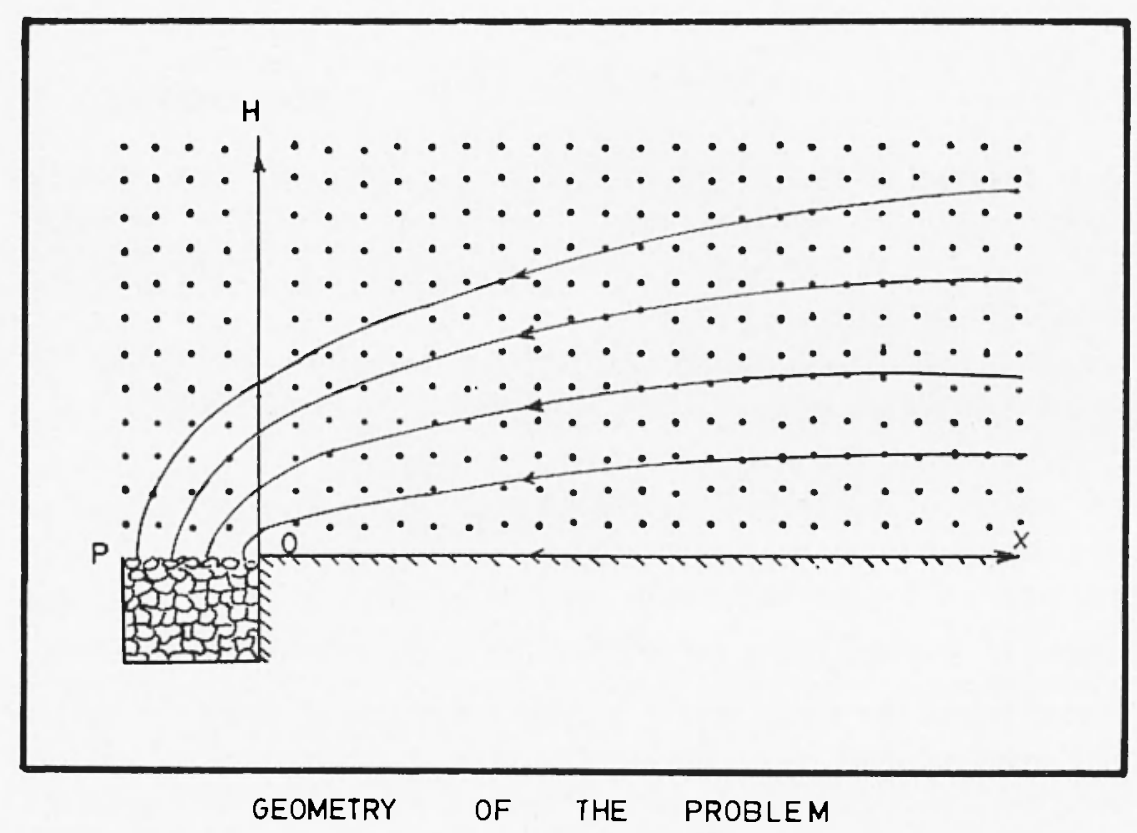

Fig. 1

4. - Solution

Using the substitution $v=\frac{a}{b} \xi$ and $F=a(1+\xi)$, equation [2] becomes:

$\frac{\partial}{\partial \xi}\left(\frac{\xi}{1+\xi} \cdot \frac{\partial H}{\partial \xi}\right)+\frac{1}{1+\xi}\left(\frac{1}{\xi}+\frac{1}{1+\xi}\right) \frac{\partial^{2} H}{\partial \theta^{2}}=0$, 
whose solution can be obtained by Fourier's method of separation of variables, for which we put:

$$
H=\alpha(\xi) \cdot \beta(\theta)
$$

Consequently, we obtain the ordinary differential equation

$$
\beta^{\prime \prime}+\lambda^{2} \beta=0
$$

and

$$
(1+\zeta) \xi^{2} \cdot \alpha^{\prime \prime}+\xi \alpha^{\prime}-\lambda^{2}(1+2 \xi) \alpha=0,
$$

where $\lambda$ is the constant of separation of variables.

The solution of equation [5] is obviously:

$$
\beta=C_{1} \operatorname{Cos} \lambda \theta+C_{2} \operatorname{Sin} \lambda \theta
$$

Again, since $\xi=0$ is a regular singular point of equation [6], it can therefore be solved by method of Frobenius (Davis, 1952). Thus, assuming a series solution in the form:

$$
\alpha=\sum_{n=0}^{\infty} a_{n}(\xi-0)^{n+r}, \quad a_{0} \neq 0
$$

equation [6] becomes :

$$
\begin{gathered}
\left(r^{2}-\lambda^{2}\right) a_{0} \xi^{\prime}+\sum_{n=1}^{\infty}\left[\left\{(n+r)^{2}-\lambda^{2}\right\} a_{n}+\right. \\
\left.+\left\{(n+r-1)(n+r-2)-2 \lambda^{2}\right\} a_{n-1}\right] \xi^{n+r}=0 .
\end{gathered}
$$


In order that equation [9] is valid for all $\xi$ in the deleted interval $0<|\xi-0|<1$, we equate the coefficients of $\xi^{k}(k=r$, $r+1, r+2, \ldots)$ to zero. Now, equating the coefficient of $\xi^{r}$ to zero, we have the characteristic equation:

$$
r-\lambda^{2}=0, \quad a_{0} \neq 0
$$

whose roots are $\lambda$ and $-\lambda$.

Again, equating the coefficients of the higher power of $s$ in [9] to zero, we obtain the recurrence formula :

$$
\begin{gathered}
{\left[\{n+r)^{2}-\lambda^{2}\right\} a_{n}+\{(n+r-1)(n+r-2)} \\
\left.\left.-2 \lambda^{2}\right\} a_{n-1}\right]=0, n \geqslant 1 .
\end{gathered}
$$

Putting $r=\lambda$, formula [11] gives:

$$
a_{n}=\left(\frac{3 n+\zeta}{n^{2}+2 n \lambda}-1\right) a_{n-1}, n+2 \lambda \neq 0 \text { and } n \geqslant 1
$$

where $\zeta=\lambda^{2}+3 \lambda-2$. Taking $r=\lambda$ and combining [12] with [8], we obtain :

$$
\begin{aligned}
x= & a_{0} \xi^{\lambda}\left[1+\left(\frac{3+\zeta}{1+2 \lambda}-1\right) \xi+\left(\frac{6+\zeta}{4+4 \lambda}-1\right) .\right. \\
& \left(\frac{3+\zeta}{1+2 \lambda}-1\right) \zeta^{2}+\left(\frac{9+\zeta}{9+6 \lambda}-1\right) . \\
& \left.\left(\frac{6+\zeta}{4+4 \lambda}-1\right)\left(\frac{3+\zeta}{1+2 \lambda}-1\right) \xi^{3}+\ldots\right] .
\end{aligned}
$$


Next, we put $r=-\lambda$ in [11] to obtain

$$
a_{n}=\left(\frac{3 n+\gamma_{1}}{n^{2}-2 n \lambda}-1\right) a_{n-1}, n-2 \lambda \neq 0 \text { and } n \geqslant 1
$$

where $\eta=\lambda^{2}-3 \lambda-2$. Taking $r=-\lambda$ and combining [14] with [8], we get:

$$
\begin{gathered}
\alpha=a_{0} \xi^{-1 .}\left[1+\left(\frac{3+\eta_{1}}{1-2 \lambda}-1\right) \xi+\left(\frac{6+\eta}{4-4 \lambda}-1\right) .\right. \\
\left.\left(\frac{3+\eta}{1-2 \lambda}:\right)=\frac{9+\eta}{9-6 \lambda}-1\right)\left(\frac{6+\eta}{4-4 \lambda}-1\right) . \\
\left.\left(\frac{3+\eta}{1-2 \lambda}: 1\right) \xi^{3}+\ldots\right] .
\end{gathered}
$$

Since the solutions [13] and [15] corresponding to the exponents $\lambda$ and $-\lambda$ respectively, are linearly independent, the solution of equation [6]may be written as :

$$
\begin{gathered}
\alpha=c_{1}^{\prime} \xi^{\lambda} \mid 1+\left(\frac{3+\zeta}{1+2 \lambda}-1\right) \xi+\left(\frac{6+\zeta}{4+4 \lambda}-1\right) \\
\left.\left(\frac{3+\zeta}{1+2 \lambda}-1\right) \xi^{2}+\ldots\right]+C_{2}^{\prime} \bar{\zeta}^{-\lambda}\left[1+\left(\frac{3+\eta^{\prime}}{1-2 \lambda}-1\right) \cdot \xi+\right. \\
\left.\left(\frac{6+\eta_{1}}{4-4 \lambda}-1\right)\left(\frac{3+\eta}{1-2 \lambda}-1\right) \xi^{2}+\ldots\right],
\end{gathered}
$$

where $C_{1}^{\prime}$ and $C_{2}^{\prime}$ are arbitrary constants. 
Hence, using [7] and [16] in [4], the general solution of the equation [3] is expressible in the form:

$$
\begin{gathered}
H=\left(C_{1} \cos \lambda \theta+C_{2} \sin \lambda \theta\right) \mid C_{1}^{\prime} \xi^{\prime} ! 1+\left(\frac{3+\xi}{1+2 \lambda}-1\right) \dot{\xi}+ \\
\left.+\left(\frac{6+\zeta}{4+4 \lambda}-1\right)\left(\frac{3+\zeta}{1+2 \lambda}-1\right) \xi^{2}+\ldots\right\} \\
+C_{2}^{\prime} \xi^{-\lambda}\left\{1+\left(\frac{3+\eta}{1-2 \lambda}-1\right) \xi+\left(\frac{6+\eta}{4-4 \lambda}-1\right) .\right. \\
\left.\left(\frac{3+\gamma_{1}}{1-2 \lambda}-1\right) \xi^{2}+\ldots\right\} \mid
\end{gathered}
$$

where the constants $C_{1}, C_{2}, C_{1}^{\prime}$ and $C_{2}^{\prime}$ are to be determined by the prescribed boundary conditions consistent with the present problem.

Applying the boundary conditions along OX, namely $\frac{\partial H}{\partial \theta}-0$ and $\theta=0$, we obtain $C_{2}=0$. Hence [17] becomes:

$$
\begin{gathered}
H=\left\lfloorC _ { 3 } \dot { \xi } ^ { \prime } \left\{ 1+\left(\frac{3+\zeta}{1+2 \lambda}-1\right) \vec{\xi}+\left(\frac{6+\zeta}{4+4 \lambda}-1\right)\right.\right. \\
\left.\left.\left(\frac{3+\zeta}{1+2 \lambda}-1\right) \check{\zeta}^{2}+\ldots\right\}+C_{4} \breve{\zeta}^{-\lambda}\right\} 1+\left(\frac{3+\eta_{1}}{1-2 \lambda}-1\right) \cdot \xi+ \\
\left.\left.+\left(\frac{6+\eta}{4-4 \lambda}-1\right)\left(\frac{3+\eta_{1}}{1-2 \lambda}-1\right) \check{\zeta}^{2}+\ldots\right\}\right] \cdot \cos \lambda \boxminus \cdot[18]
\end{gathered}
$$


At the head reservoir, where the flow is laminar, let $\xi=\xi_{1}$. Then, using the boundary conditions $\theta=0$ and $H=H_{0}$ in [18], we get:

$$
C_{3}=\frac{H_{0}-C_{4} \xi_{1}-\lambda\left\{1+\left(\frac{3+\eta}{1-2 \lambda}-1\right) \xi_{1}+\left(\frac{6+\eta}{4-4 \lambda}-1\right)\left(\frac{3+\eta}{1-2 \lambda}-1\right) \xi_{1}^{2}+\ldots\right\}}{\xi_{1}^{\lambda}\left\{1+\left(\frac{3+\zeta}{1+2 \lambda}-1\right) \xi_{1}+\left(\frac{6+\zeta}{4+4 \lambda}-1\right)\left(\frac{3+\zeta}{1+2 \lambda}-1\right) \xi_{1}^{2}+\ldots\right\}}[
$$

which on substituting in [18] gives:

$$
\begin{aligned}
& \operatorname{Cos} \lambda \theta
\end{aligned}
$$

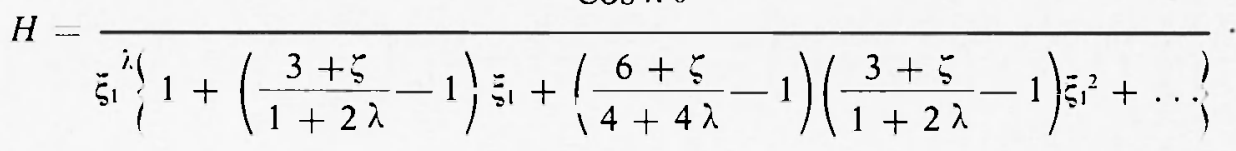

$$
\begin{aligned}
& {\left[H_{0} \xi^{\lambda} \hat{i}_{1}+\left(\frac{3+\zeta}{1+2 \lambda}-1\right) \xi\left(\frac{6+\zeta}{4+4 \lambda}-i\right)\left(\frac{3+\zeta}{1+2 \lambda}-i\right) \xi^{2}+\ldots\right]} \\
& \left.\left.+C\left(\frac{\xi_{1}}{\xi}\right) \cdot 1+\left(\frac{3+\zeta}{1+2 \lambda}-1\right)=1 \frac{6+\zeta}{(4+4 \lambda}-1\right)\left(\frac{3+\zeta}{1+2 \lambda}-1\right) \xi_{1}{ }^{2}+\ldots\right] . \\
& \cdot\left[1+\left(\frac{3+\eta}{1-2 \lambda}-1\right) \vdots\left(\frac{6+\eta}{4-4 \lambda}-1\right)\left(\frac{3+\eta}{1-2 \lambda}-1\right), \xi^{2}+\ldots\right] \\
& -\left(\frac{\dot{\xi}}{\dot{\xi}_{1}}\right)^{\lambda}\left[1+\left(\frac{3+\eta}{1-2 \lambda}-1\right) \xi_{1}+\left(\frac{6+\eta}{4-4 \lambda}-1\right)\left(\frac{3+\eta}{1-2 \lambda}-1\right) \vec{\xi}_{1}{ }^{2}+\ldots\right] \\
& \left.\left.\cdot\left[1+\left(\frac{3+\zeta}{1+2 \lambda}-1\right) \xi+\left(\frac{6+\zeta}{4+4 \lambda}-1\right)\left(\frac{3+\zeta}{1+2 \lambda}-1\right) \xi^{2}+\ldots\right]\right\}\right]
\end{aligned}
$$


Lastly, at the drainage slit $O P$, where the flow is almost turbulent, let $\xi=\varsigma_{2}$. Applying the corresponding boundary conditions $H=0$ and $\theta=\pi / 2$ in [20], we have:

$$
\begin{aligned}
& H_{0} \xi_{2}{ }^{\lambda}\left(1+\left(\frac{3+\zeta}{1+2 \lambda}-1\right) \xi_{2}+\left(\frac{6+\zeta}{4+4 \lambda}-1\right)\left(\frac{3+\zeta}{1+2 \lambda}-1\right) \xi_{2}{ }^{2}+\ldots\right\} \\
& C_{4}= {\left[\left(\frac{\xi_{2}}{\xi_{1}}\right)^{\lambda}\left\{1+\left(\frac{3+\eta}{1-2 \lambda}-1\right) \xi_{1}+\left(\frac{6+\eta}{4-4 \lambda}-1\right)\left(\frac{3+\eta}{1-2 \lambda}-1\right) \xi_{1}{ }^{2}+\ldots\right\}\right.} \\
& \cdot\left\{1+\left(\frac{3+\zeta}{1+2 \lambda}-1\right) \xi_{2}+\left(\frac{6+\zeta}{4+4 \lambda}-1\right)\left(\frac{3+\zeta}{1+2 \lambda}-1\right) \xi_{2}{ }^{2}+\ldots\right\} \\
&-\left(\frac{\xi_{1}}{\xi_{2}}\right)^{\lambda}\left\{1+\left(\frac{3+\zeta}{1+2 \lambda}-1\right) \xi_{1}+\left(\frac{6+\zeta}{4+4 \lambda}-1\right)\left(\frac{3+\zeta}{1+2 \lambda}-1\right) \xi_{1}{ }^{2}+\ldots\right\} \\
&\left.\cdot\left\{1+\left(\frac{3+\eta}{1-2 \lambda}-1\right) \xi_{2}+\left(\frac{6+\eta}{4-4 \lambda}-1\right)\left(\frac{3+\eta}{1-2 \lambda}-1\right) \xi_{2}{ }^{2}+\ldots\right\}\right)
\end{aligned}
$$

Now, combining [21] with [20] and introducing the dimensionless total head:

$$
Z(\lambda ; \vdots, \theta)=\frac{H}{H_{0}}, \lambda \neq \pm \frac{n}{2},
$$

$n$ being a positive integer, we finally obtain:

$$
Z(\lambda ; \dot{\bar{c}}, \theta)=\frac{\cos \lambda \theta}{\xi_{1}^{\lambda}\left\{1+\left(\frac{3+\zeta}{1+2 \lambda}-1\right) \vec{\xi}_{1}+\left(\frac{6+\zeta}{4+4 \lambda}-1\right)\left(\frac{3+\zeta}{1+2 \lambda}-1\right) \vec{\xi}_{1}^{2}+\ldots\right\}}
$$




$$
\begin{aligned}
& {\left[\xi^{\lambda}\left\{1+\left(\frac{3+\zeta}{1+2 \lambda}-1\right) \xi+\left(\frac{6+\zeta}{4+4 \lambda}-1\right)\left(\frac{3+\zeta}{1+2 \lambda}-1\right) \xi^{2}+\ldots\right\}+\right.} \\
& \xi_{2}^{\lambda}\left\{1+\left(\frac{3+\zeta}{1+2 \lambda}-1\right) \xi_{2}+\left(\frac{6+\zeta}{4+4 \lambda}-1\right)\left(\frac{3+\zeta}{1+2 \lambda}-1\right) \xi_{2}{ }^{2}+\ldots\right\} \\
& +\frac{\left(\frac{\xi_{2}}{\xi_{1}}\right)^{\lambda}\left\{1+\left(\frac{3+\eta}{1-2 \lambda}-1\right) \xi_{1}+\left(\frac{6+\eta}{4-4 \lambda}-1\right)\left(\frac{3+\eta}{1-2 \lambda}-1\right) \xi_{1}^{2}+\ldots\right\}}{x} \times \\
& \cdot\left\{1+\left(\frac{3+\zeta}{1+2 \lambda}-1\right) \xi_{2}+\left(\frac{6+\zeta}{4+4 \lambda}-1\right)\left(\frac{3+\zeta}{1+2 \lambda}-1\right) \xi_{2}{ }^{2}+\ldots\right\} \times \\
& -\left(\frac{\xi_{1}}{\xi_{2}}\right)^{\lambda}\left\{1+\left(\frac{3+\zeta}{1+2 \lambda}-1\right) \xi_{1}+\left(\frac{6+\zeta}{4+4 \lambda}-1\right)\left(\frac{3+\zeta}{1+2 \lambda}-1\right) \xi_{1}^{2}+\ldots\right\} . \\
& \left.\cdot\left\{1+\left(\frac{3+\eta}{1-2 \lambda}-1\right) \xi_{2}+\left(\frac{6+\eta}{4-4 \lambda}-1\right) \quad\left(\frac{3+\eta}{1-2 \lambda}-1\right) \xi_{2}{ }^{2}+\ldots\right\}\right] . \\
& \times\left\{\left(\frac{\xi_{1}}{\xi}\right)^{\lambda}\left[1+\left(\frac{3+\zeta}{1+2 \lambda}-1\right) \xi_{1}+\left(\frac{6+\zeta}{4+4 \lambda}-1\right)\left(\frac{3+\zeta}{1+2 \lambda}-1\right) \dot{\xi}_{1}{ }^{2}+\ldots\right] .\right. \\
& \cdot\left[1+\left(\frac{3+\eta}{1-2 \lambda}-1\right) \xi+\left(\frac{6+\eta}{4-4 \lambda}-1\right)\left(\frac{3+\eta}{1-2 \lambda}-1\right) \xi^{2}+\ldots\right] \times \\
& -\left(\frac{\xi}{\xi_{1}}\right)^{\lambda}\left[1+\left(\frac{3+\eta}{1-2 \lambda}-1\right) \xi_{1}+\left(\frac{6+\eta}{4-4 \lambda}-1\right)\left(\frac{3+\eta}{1-2 \lambda}-1\right) \xi_{1}^{2}+\ldots\right] . \\
& \left.\left.\cdot\left[1+\left(\frac{3+\zeta}{1+2 \lambda}-1\right) \xi+\left(\frac{6+\zeta}{4+4 \lambda}-1\right)\left(\frac{3+\zeta}{1+2 \lambda}-1\right) \xi^{2}+\ldots\right]\right\}\right] \\
& \vec{\xi}_{1} \leqslant \dot{\xi} \leqslant \xi_{2}, 0<|\xi-0|<1 . \\
& \text {... [23] }
\end{aligned}
$$


Equation [23] represents the free surface corresponding to a steady state, two-dimensional general flow system, where in an incompressible fuid moves through undeformable porous media from an infinitely large distance into a drainage slit.

\section{5. - Discussion}

The general equation [23] of free surface expresses the dimensionless total head $Z$ as a function of $\lambda, \xi$ and $\theta$. The parameter $\lambda$ is the constant of the separation of variables $\bar{\xi}$ and $\theta$. By assigning any value to the parameter $\lambda$ such that $\lambda \neq \pm n / 2$ ( $n$ being a positive integer), $Z$ can be represented as a function of $\bar{\xi}$ and $\theta$ alone.

To get a definite idea of the result [23], we restrict the value of $\bar{\xi}$ such that $\bar{\xi}_{1} \leqslant \xi \leqslant \bar{\xi}_{2}$ and $0<\xi<1$, where $\vec{\xi}_{1}$ and $\bar{\zeta}_{2}$ are the Reynolds numbers corresponding to laminar and turbulent types of flow, respectively. Under these conditions, appropriate series solutions representing $Z$ as a function of $\theta$ can be obtained for different values of $\zeta$. Further, to analyse how the nature of free surface depends on $\bar{\xi}, Z$ can be plotted against $\xi$ for different values of $\theta$.

\section{6. - Numerical, EXAMPle}

Now to illustrate [23] numerically, we take $\lambda=1.2, \varsigma_{1}=0.01$ and $\bar{\xi}=1.0$. Approximate values of $Z(1.2 ; \bar{\xi}, \theta)$, corresponding to $\theta=\pi / 6, \pi / 4$ and $\pi / 3$ have been graphically represented in Fig. 2 for values of $\xi$ starting from 0.05 . From Fig. 2 it is seen that $Z$ increases as $\xi$ increases for fixed values of $\theta$. Further, it is also observed that $Z$ decreases as $\theta$ increases comparatively for all values of $\xi$.

Again, approximate values of $Z(1.2 ; \xi, \theta)$ corresponding to $\bar{\xi}=0.02,0.07,0.50$ and 0.90 have been graphically represented in Fig. 3 for values of $\theta$ starting from $\pi / 18$. From Fig. 3 it is seen that $Z$ decreases as $\theta$ increases for fixed values of $\dot{\xi}$. It is also 


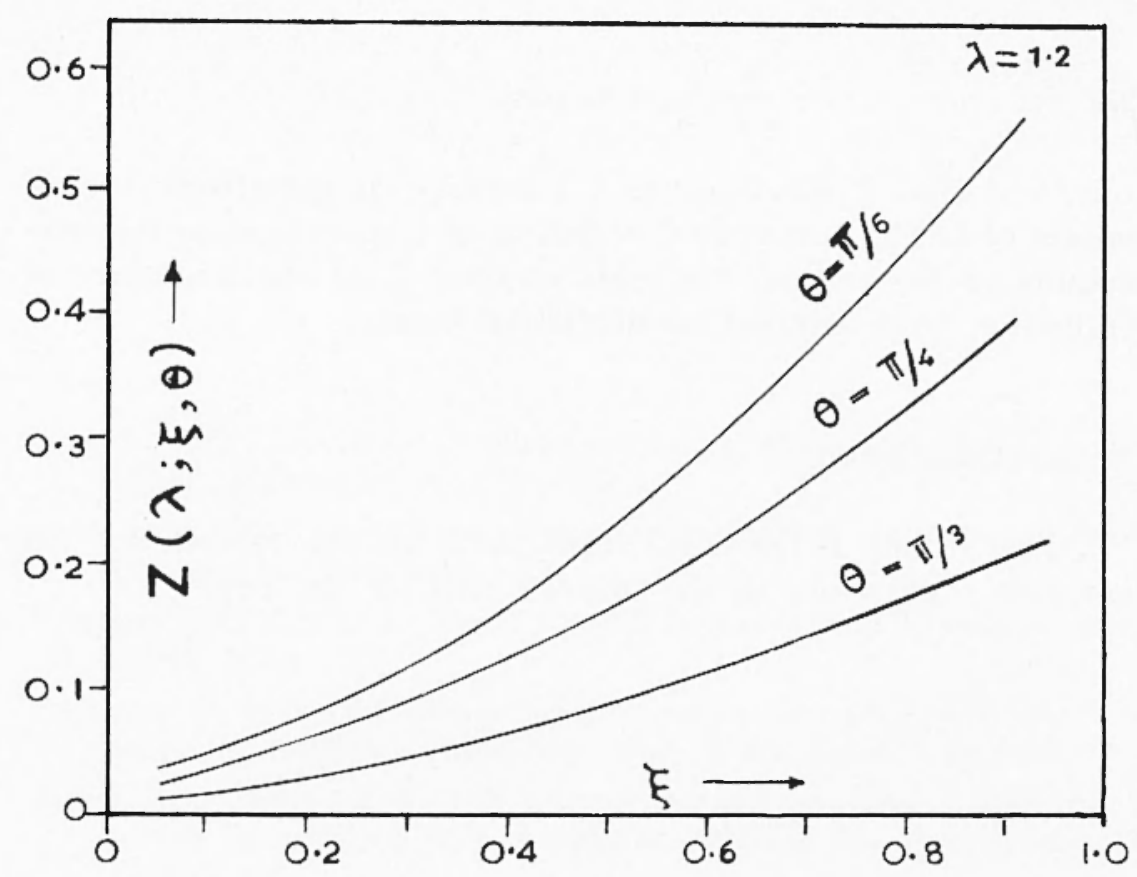

Fig. 2

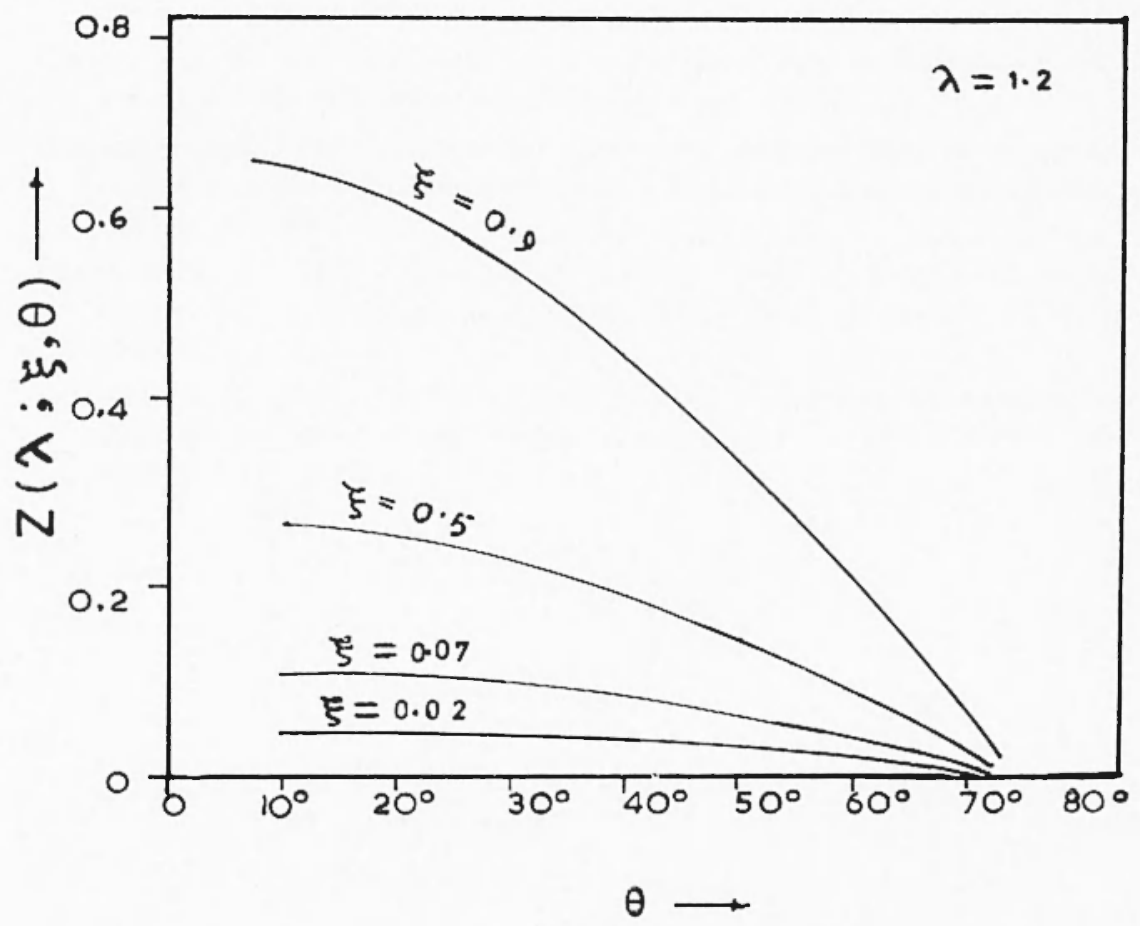

Fig 3 
observed that $Z$ increases as $\xi$ increases comparatively for all values of $\theta$. The curve for $\varsigma=0.07$ is of importance, as it corresponds to the critical Reynolds number $\bar{\xi}_{c}$ at the boundary of transition from laminar to non-linear laminar.

\section{ACKNOWLEDGEMENT}

The author is very much thankful to the referee for his valuable suggestions in the improvement of the paper. 


\section{R E FE REN C E S}

BEAR J., 1972 - Dynamics of fluids in porous media. American Elsevier Publishing Company Inc., New York.

Davis C. V., 1952 - Hand book of Applied Hydraulics. Mc Graw-Hill Book Company Inc., New York.

EllenbaAs J. R., Katz D. L., 1948. - A radial turbulent flow formula. "Trans. AIME », 174, p. 25.

ENGELUND F., 1953 - On laminar and turbulent flow of ground water in homogeneous sand. "Trans. Dan. Acad. Teach. Sci. ”, 3, pp. 1-105.

JaIN S. K., UPADHYAY K. K., 1976 - Non-Linear laminar flow of fluid into a partially penetrating well. "Gerlands Beiträge Zur Geophysik», 85, 2, pp. 151-156.

Scheidegger A. E., 1960 - The Physics of flow through porous media. Mac Millan Company, New York.

UPADHYAY K. K., 1975 - Non-linear laminar flow into eccentrically placed well. "Annali di Geofisica ", XXVIII, 4, pp. 311-319.

UpaDHYAY, K. K., 1977 - Non-linear flow into well partially penetrating a porous aquifer of finite thickness. "Pure and Applied Geophysics", 115, pp. 631-638.

UPADHYAY K. K., 1977 - Non-linear laminar flow of fluid into a fully penetrating cylindrical well. "Ind. Jour. Theo. Physics", 25, 4, pp. 149-156.

UpadHYAY K. K., 1978 - Turbulent flow of fluid into a homogeneous porous medium on an inclined bedrock. "Rev. Roum. Sci. Techn - Méc. Appl. ", 23, 3, pp, 353 - 357. 